\title{
Diferencias en las amplitudes articulares entre varones y mujeres en edad escolar
}

\author{
David Ramos Espada a , José Luis González Montesinos ${ }^{\mathrm{b}}$ y Jesús Mora Vicente
}

aProfesor I.E.S. Obispo Argüelles. Villablino. León. España.

'Facultad de Ciencias de la Educación. Universidad de Cádiz. Cádiz. España.

\section{RESUMEN}

Introducción: En el presente estudio se han analizado las diferencias existentes entre hombres y mujeres, en relación a la amplitud articular a lo largo de la vida del escolar.

Métodos: Se analiza una población de 420 alumnos y alumnas, con edades comprendidas entre los 7 y los 17 años (Educación Primaria y Educación Secundaria Obligatoria). El estudio, se realiza mediante la aplicación de una batería de tests de valoración de la flexibilidad por medio de mediciones goniométricas. Esta batería consta de 10 pruebas, de las que se extraen datos relativos a la movilidad articular de hombros, cadera, rodilla y tobillos.

Resultados: Los resultados obtenidos indican que la amplitud articular de los sujetos estudiados disminuye con la edad. Asimismo estos resultados son mejores en las mujeres que en los hombres, con diferencias significativas en tres de las pruebas incluidas en el test. Los picos de acortamientos varían en función del sexo del sujeto.

PALABRAS CLAVE: Flexibilidad. Desequilibrios musculares. Músculos posturales. Educación Física. Goniómetro.

\begin{abstract}
Introduction: The present study analyzed differences between boys and girls in articular width throughout school life. Methods: We analyze a population of 420 boys and girls aged between 7 and 17 years old (primary and secondary education).

A battery of tests evaluating flexibility through goniometric measurements was carried out. This battery consisted of 10 tests, from which data related to the articular mobility of the shoulders, hip, knee and ankles were gathered.

Results: The results obtained indicate that articular width in the subjects studied decreases with age. The results were better in girls than in boys, with substantial differences in three of the elements included in the test. Peak reductions varied, depending on sex.
\end{abstract}

KEY WORDS: Flexibility. Muscular imbalances. Postural muscles. Physical Education. Goniometry.

\section{INTRODUCCIÓN}

Uno de los factores determinantes de la flexibilidad es el sexo de la persona. La mujer, por norma general, obtiene mejores valores de flexibilidad que el varón. Para algunos autores, esto se debe a una mayor presencia de estrógeno circulante, un porcentaje más elevado de tejido adiposo, un menor porcentaje de masa muscular y, lo que es más determinante, una mayor producción de relaxina ${ }^{1}$.

Hubley (citado por Mac Dougall ${ }^{2}$ ) define "flexibilidad" como la amplitud de movimiento de una sola articulación o de una serie de articulaciones y refleja la capacidad de las unidades musculotendinosas para elongarse tanto como se lo permi- tan las restricciones físicas de la articulación. Otra definición que viene a completar la anterior, sería la aportada por $\mathrm{Pila}^{3}$ como la capacidad de amplitud de un movimiento en un segmento articular determinado, facultad que puede verse afectada tanto por la capacidad de elongación de los distintos tejidos que constituyen una articulación, como por la morfología anatómica de la misma, y que pueden tener una causa genética o patológica.

Otros componentes importantes para delimitar el término de flexibilidad son la elasticidad, la elongación y la laxitud. La flexibilidad puede ser estática o dinámica; esta última es difícil de definir, y se asocia con la oposición o resistencia al movi- 
miento de las articulaciones ${ }^{4}$. La gran mayoría de autores se refieren a la flexibilidad como a una medida estática.

\section{Características principales de la flexibilidad}

- La flexibilidad es específica de la articulación y de la acción de la articulación (Harris, 1969; Hupprich, 1950; Leighton, 1957; Munroe, y Romace, 1975; citados por Mac Dougall²).

- La flexibilidad es la capacidad que permite realizar movimientos de gran amplitud. Es una capacidad involutiva, lo que significa que el individuo nace dotado con una gran flexibilidad, que progresivamente va perdiendo (Fernández, citado por Santonja $\left.a^{5}\right)$.

Para Arregui y Martínez 4 , la complejidad del estudio de la flexibilidad se debe a múltiples factores, como son la capacidad de las unidades musculotendinosas para estirarse, las restricciones físicas de cada articulación, el sexo, la edad, el nivel de crecimiento, la práctica deportiva y el entrenamiento.

El ser humano tiene dos tipos de músculos: estáticos o posturales, normalmente muy tonificados, que permiten la posición erguida y la bipedestación, y dinámicos o fásicos, normalmente poco tónicos, que realizan los movimientos de gran amplitud y que están relacionados con la locomoción ${ }^{6}$. Los primeros representan las dos terceras partes de nuestra musculatura y suelen soportar un trabajo continuado como es la adopción de posturas estáticas durante largos períodos de tiempo. En situaciones de sobrecarga o de estrés, la musculatura estática evoluciona siempre hacia el acortamiento. Sin embargo, la musculatura dinámica tiende al relajamiento y a la debilidad, sobre todo en personas sedentarias o que realizan poca actividad física. Si aparece un falta de amplitud articular, se deberá a la rigidez de los músculos estáticos.

La amplitud de movimiento de una articulación está limitada por varios factores, entre los que destacan las propias estructuras óseas que forman la articulación, el cartílago articular y los tejidos blandos que la rodean: músculos, tendones, fascia, ligamentos y piel (Johns y Wright, 1962, citado por Mac Dougall²).

Las ganancias de flexibilidad deben ir encaminadas a la mejora de la extensión de la unidad musculotendinosa. Toda actividad física solicita de forma indiferenciada los músculos estáticos y dinámicos. Esto ejerce un papel beneficioso sobre los músculos dinámicos, que tienden a la hipotonicidad, pero en su contra tiene un efecto que acentúa la hipertonía y rigidez de los estáticos.

La evolución de la flexibilidad varía de forma compleja a lo largo de la etapa escolar. No todas las articulaciones varían sus amplitudes por igual: algunas disminuyen más que otras e incluso se producen aumentos.
La flexibilidad se debe trabajar de forma continua e incidiendo en las partes tendentes al acortamiento. Este trabajo permite prevenir posibles lesiones derivadas de estos acortamientos y debe ser incluido en los calentamientos que han de efectuar los alumnos y las alumnas. La finalidad será disponer de forma adecuada al aparato locomotor para la realización de cualquier actividad física.

Un concepto muy relacionado con la flexibilidad y la amplitud articular es el de acortamiento y desequilibrio muscular. Para Janda (citado por Liebenson7), "la base para la mayoría de los desequilibrios musculares proviene de nuestra previsible respuesta a las exigencias estresantes ambientales". En el caso que nos ocupa, las posturas forzadas en las aulas, las tareas repetitivas, la tensión de la gravedad y la inactividad son algunas de las causas que degeneran en tal situación.

Los músculos posturales de los escolares tienen tendencia hacia el sobreuso y hacia el acortamiento eventual, mientras que los músculos fásicos tienden hacia el desuso y la debilidad. Según Liebenson ${ }^{7}$, estos músculos están agrupados con frecuencia como antagonistas emparejados y parecen estar afectados por la ley de Sherrington de la inhibición recíproca. Así, si un músculo postural como el psoas ilíaco se acorta por sobreuso, no sólo limitará mecánicamente el alcance de los movimientos de su antagonista, el glúteo mayor, sino que también inhibirá neurológicamente su acción. Esta combinación de influencias biomecánicas y neurofisiológicas es un fuerte estímulo para la creación y el mantenimiento de desequilibrios musculares en los escolares. La expresión desequilibrio muscular describe la situación en la cual algunos músculos se inhiben y debilitan, mientras otros quedan "apretados" (acortados) y pierden su extensibilidad. Los músculos moderadamente "apretados" (acortados) suelen ser más fuertes de lo normal, aunque en el caso de tensión pronunciada se produce alguna reducción de la fuerza muscular. Esto se denomina debilidad de tensión (Janda, citado por Liebenson ${ }^{7}$ ).

El tratamiento de la tensión no radica en el fortalecimiento, que incrementaría la tensión y provocaría una mayor debilidad, sino en el estiramiento, con la intención de influir, no en el tejido conectivo no contráctil del músculo, sino en el retráctil.

El desequilibrio muscular no queda limitado a ciertas partes del cuerpo, sino que gradualmente afecta a todo el sistema muscular estriado. Puesto que el desequilibrio muscular suele preceder la aparición de síndromes de dolor, una evaluación completa puede ayudar a introducir medidas preventivas ${ }^{8}$.

Las consecuencias del desequilibrio muscular son las siguientes (Janda, citado por Liebenson ${ }^{7}$ ): 
- Alteración de los mecanismos articulares (distribución desigual de la presión).

- Amplitud limitada de movimiento e hipermovilidad compensatoria.

- Cambio en la entrada propioceptiva.

- Inhibición recíproca deteriorada.

- Programación alterada de modelos de movimiento.

Cuando la musculatura tónica y fásica no están compensadas hay un desequilibrio muscular. Los músculos acortados están duros y no tienen elasticidad en la fase de relajación, por ello se fatigan y producen sobrecargas dolorosas. Así pues, una vida sedentaria provoca el sobreuso de los músculos posturales, favoreciendo así el desarrollo de la rigidez. Simultáneamente, los músculos fásicos o dinámicos tienden a debilitarse por el desuso.

\section{Objetivos del estudio}

Los objetivos del estudio son los siguientes:

- Estudiar las diferencias existentes entre los varones y las mujeres en relación a la amplitud articular lo largo de la vida del escolar (educación primaria y educación secundaria).

- Incidir en la importancia que tiene la flexibilidad como parte importante en el trabajo diario con los alumnos en las clases de educación física.

\section{MATERIAL Y MÉtodo}

\section{Población y ámbito de estudio}

La población utilizada para este estudio fue de un total de 420 alumnos y alumnas $(n=420)$ pertenecientes a diversos centros de educación primaria y educación secundaria obligatoria. Los centros en los que se realizó el estudio, pertenecientes a la localidad de Ponferrada (León), fueron los siguientes:

- Colegio público Flores del Sil.

- Colegio público comarcal Virgen de la Encina.

- Instituto de educación secundaria Álvaro de Mendaña.

- Instituto de educación secundaria Virgen de la Encina.

Las edades de los sujetos varían entre los 7 y los 17 años. La distribución de alumnos por edades es del $7 \%$ para los sujetos con 7, 10, 15 y 16 años, del $8 \%$ para los de 8 años, del $11 \%$ para los sujetos de 9, 12 y 14 años, del $12 \%$ para las edades de 11 y 13 años y del 6\% para los sujetos con 17 años.
Su distribución por sexos es del 51\% para los varones y del $49 \%$ para las mujeres.

\section{Metodología}

Instrumentos

El estudio efectuado mediante la aplicación de una batería de test con el fin de comprobar la evolución de la amplitud articular y la flexibilidad, se realiza con mediciones goniométricas. La batería consta de 10 pruebas, en 9 de las cuales se extraen datos relativos a la flexibilidad.

Las mediciones fueron realizadas con materiales asequibles y sencillos, tanto en el aspecto económico como de utilización por parte del examinador, con la finalidad de que este test pueda ser utilizado por el profesorado que imparte la asignatura de educación física.

Las pruebas realizadas evalúan principalmente los músculos que tienden al acortamiento (músculos posturales).

\section{Descripción del test de valoración de las amplitudes articulares}

Para la medición de los posibles acortamientos y del déficit de flexibilidad se utilizaron una serie de ítems reunidos en una batería que mide la amplitud articular del hombro, de la cadera, de la rodilla y del tobillo. Los test se basan en mediciones goniométricas, siguiendo los protocolos propuestos por los diferentes autores consultados. La batería consta de las siguientes pruebas:

1. Valoración de la articulación del hombro

- Prueba de rotadores internos y aductores del hombro (RIAH). En posición de decúbito supino, rodillas en flexión, con las manos detrás del cuello, descansando la columna lumbar lo más plana posible y los codos apoyados sobre el suelo sin tensión. La presencia de cifosis impide realizar la prueba. Se debe anotar el contacto o no de los codos en el suelo. Asimismo, y para comprobar posibles descompensaciones, se deben anotar diferencias entre el lado derecho e izquierdo' (fig. 1A).

- Prueba de Kendall (PK). Con esta prueba se evalúa la movilidad de los aductores del hombro. Sin acortamiento de los aductores y rotadores internos del hombro, la articulación del hombro puede ser flexionada completamente mientras la porción inferior de la espalda está aplicada sobre la camilla o suelo. Con acortamiento de los aductores y rotadores internos del hombro, la 
TRABAJOS ORIGINALES

Figura I

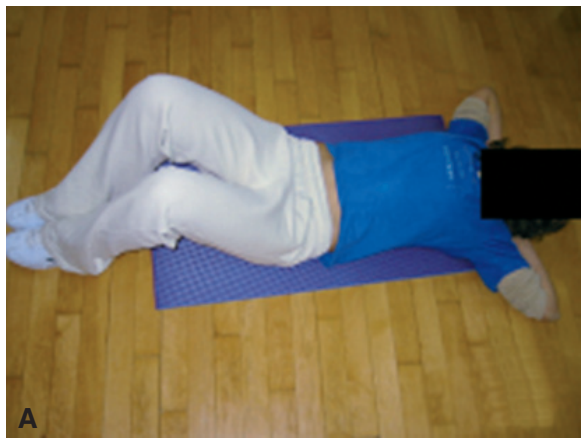

A) Prueba de rotadores internos y aductores de hombro. B) Prueba de Kendall. C) Diagonal posterior.
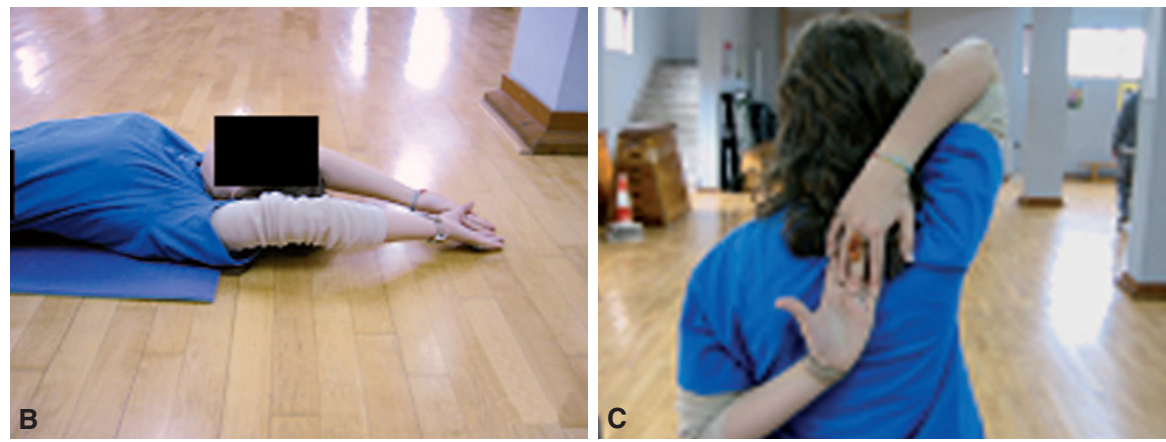

articulación del hombro no puede ser flexionada completamente con la porción de la espalda aplanada. Esto indica un posible acortamiento del dorsal ancho, del pectoral mayor y del redondo mayor. Se considera angulación normal $180^{\circ}$, es decir, articulación escapulohumeral y húmero en contacto con el suelo. El movimiento es realizado de forma activa por el sujeto ${ }^{10}$ (fig. 1B).

- Diagonal posterior (DP). En bipedestación, con los brazos por detrás de la espalda, uno de ellos llevado por la zona dorsal de la espalda, y el otro por la zona lumbar. Anotar el contacto o no de las manos, con distinción del lado derecho e izquierdo. Se mide el lado del brazo que pasa hacia atrás por la zona dorsal. Su objetivo es conocer los desequilibrios y disimetrías de la cintura escapular ${ }^{11}$ (fig. 1C).

\section{Valoración de la musculatura isquiotibial (fig. 2)}

- Flexión de cadera con rodilla en extensión (FCRE). En posición de decúbito supino, con los brazos rectos y colocados a los lados del tronco, flexionar lo máximo posible la cadera sin flexionar la rodilla. La extremidad opuesta, que ayuda a evitar el movimiento bascular posterior de la pelvis, debe permanecer en contacto con el banco. Se mide el ángulo formado por ambas extremidades. La pierna que está en contacto con el banco se inmovilizará con una eslinga o un compañero, para evitar la flexión de la rodilla y la basculación pélvica, y así facilitar la medición al examinador. El goniómetro se colocará con un brazo paralelo al banco (dispone de una burbuja de nivel) y el otro eje coincidiendo con trocánter mayor del fémur y la rodilla ${ }^{12}$.

\section{Valoración del músculo psoas ilíaco y el recto anterior}

- Prueba de Thomas (PTh) ${ }^{7}$. El sujeto se coloca encima de la camilla o del plinton, tendido en decúbito supino, y a conti-

\section{Figura 2 Prueba de flexión de cadera con rodilla en extensión.}

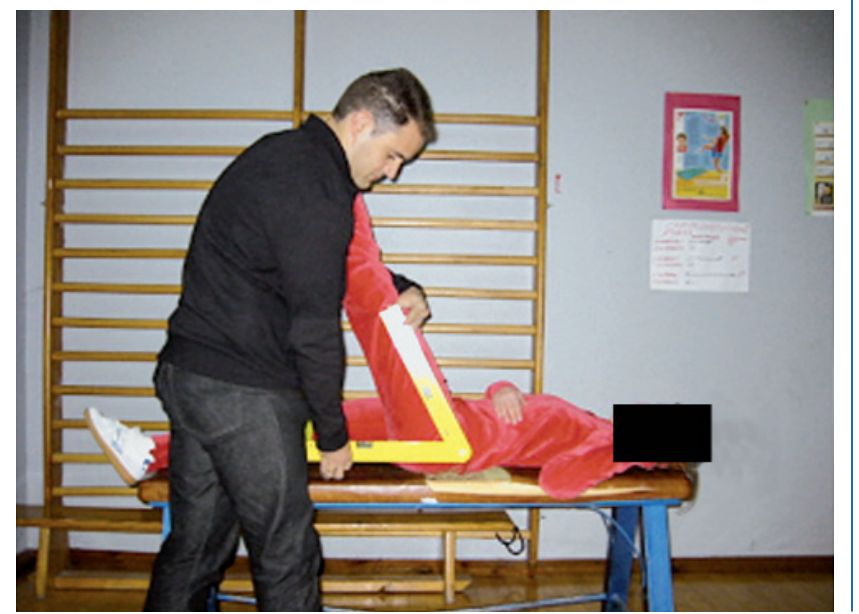

nuación coge con sus manos una de sus piernas por la rodilla y la acerca al pecho. La pierna correspondiente a la cadera que se va a examinar fuera de camilla. Si existe acortamiento del psoas ilíaco, se producirá elevación de la pierna de apoyo en mesa. La cadera será incapaz de extenderse más allá de los 10 o $15^{\circ}$ considerados normales. Si existe acortamiento del recto anterior, se producirá extensión de la rodilla de apoyo en mesa, y acortamiento si la extensión de la rodilla es superior a $125^{\circ 13}$ (fig. 3).

4. Rotadores de cadera internos y externos (RCI y RCE) (fig. 4)

- En posición sedente sobre una superficie elevada, con la pelvis estabilizada, y mediante movilización pasiva, se mide la flexibilidad de los rotadores externos con una rotación interna 
TRABAJOS ORIGINALES

Figura $3 \int$ Prueba de psoas ilíaco.

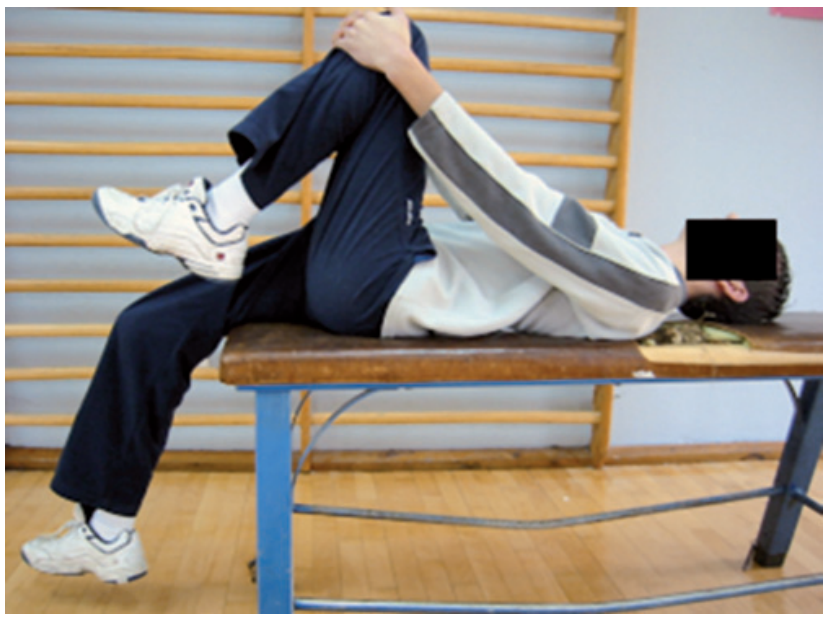

de la cadera. Posteriormente se mide la flexibilidad de los rotadores internos realizando una rotación externa de la cadera. Se mide el ángulo formado por la pierna y la perpendicular del suelo a la rótula ${ }^{12}$.

\section{Aductores de cadera $(\mathrm{AC})^{13}$}

- Decúbito supino con un eje del goniómetro sobre ambas crestas ilíacas de la cadera y el otro eje hacia la rodilla de la pierna de medición. Se abducirá al máximo la cadera que se mide. Se medirán las dos piernas (fig. 5).

6. Elongación de los flexores plantares $(\mathrm{EFP})^{7}$

- El sujeto sentado con las rodillas extendidas realiza una flexión del tobillo (movilización activa). La rodilla no ha de es-
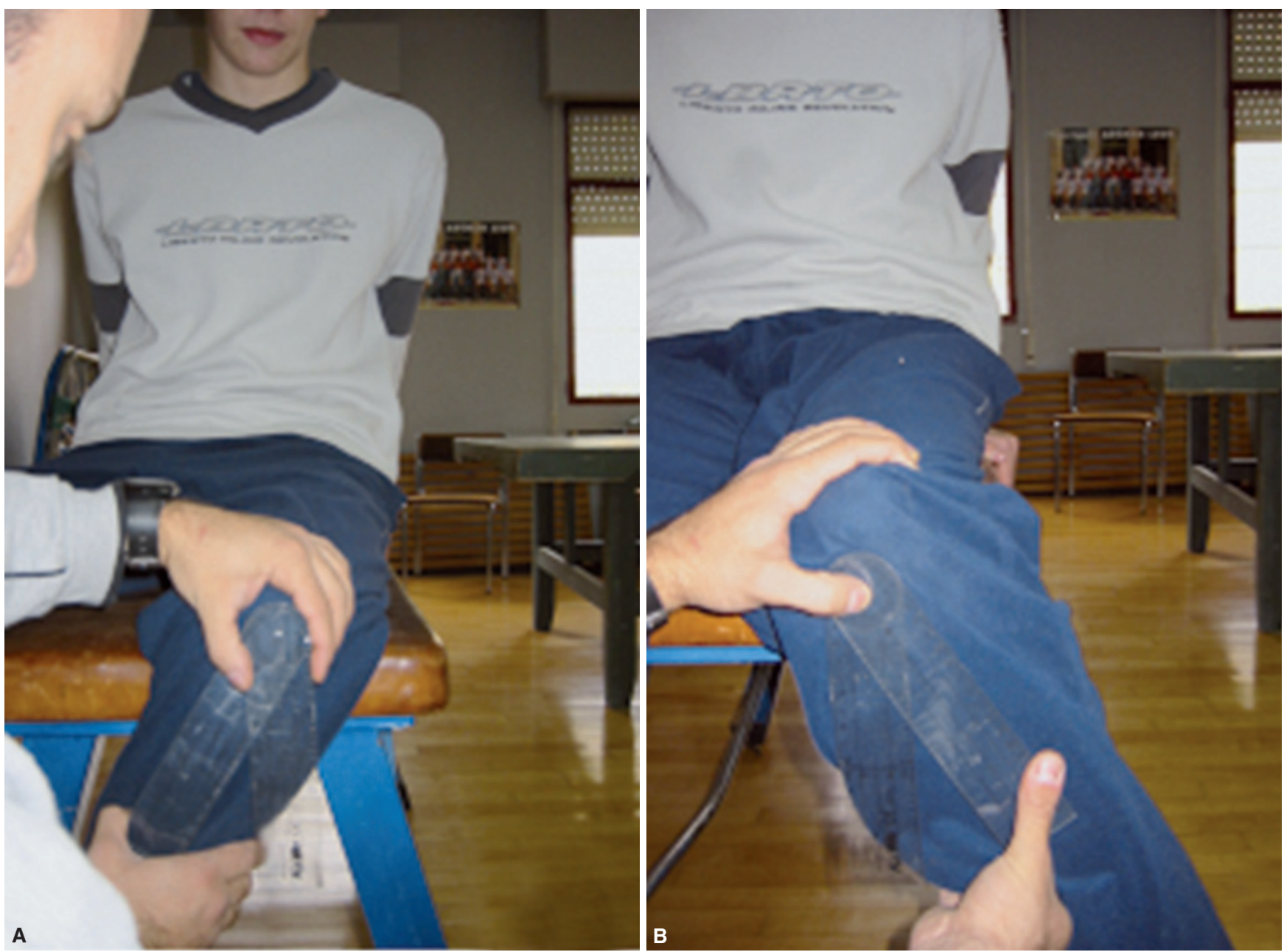
TRABAJOS ORIGINALES

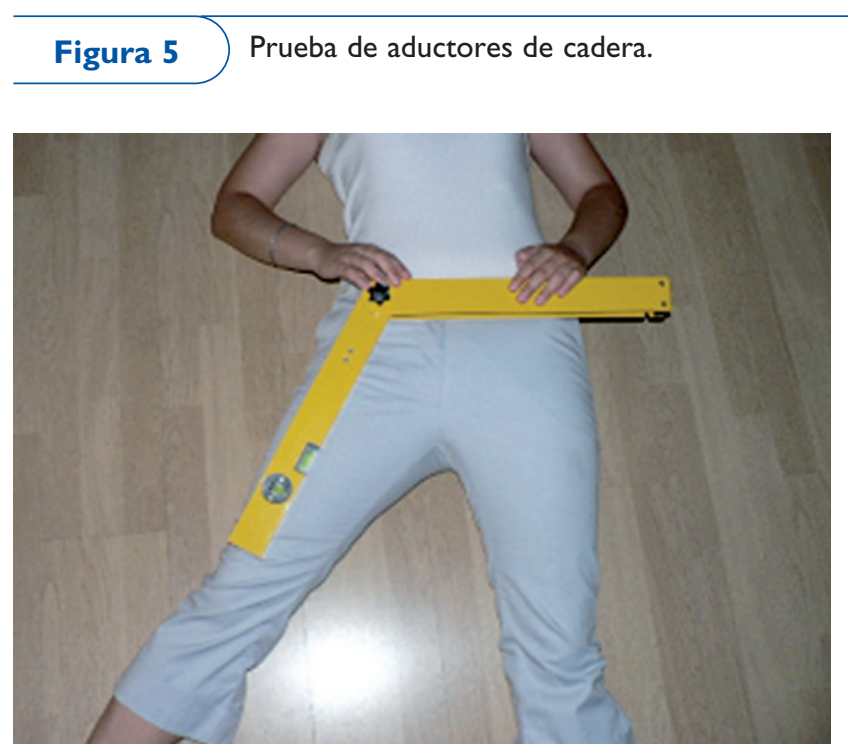

\section{Figura 6 Prueba de elongación de los flexores plantares.}

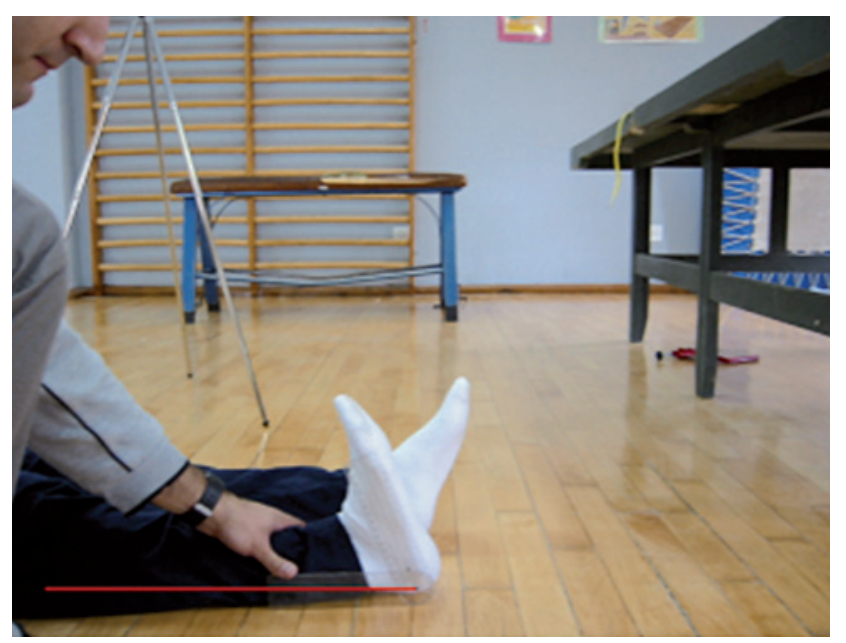

tar en flexión, debido a que se eliminaría la tensión de los gemelos y así los datos sólo expresarían el rango de movilidad articular sin intervención del componente muscular. Se considera normal la medición de 10 a $15^{\circ}$ (fig. 6).

\section{Valoración de la musculatura del cuádriceps}

- Prueba de Nachlas (PN) y prueba de Ridge (PR $)^{13}$. En posición decúbito prono sobre la esterilla, se flexiona de forma pasiva la rodilla y se mide el ángulo antes de que la columna lumbar comience a extenderse o la cadera comience a elevarse. Se consideran mediciones normales los ángulos comprendidos entre
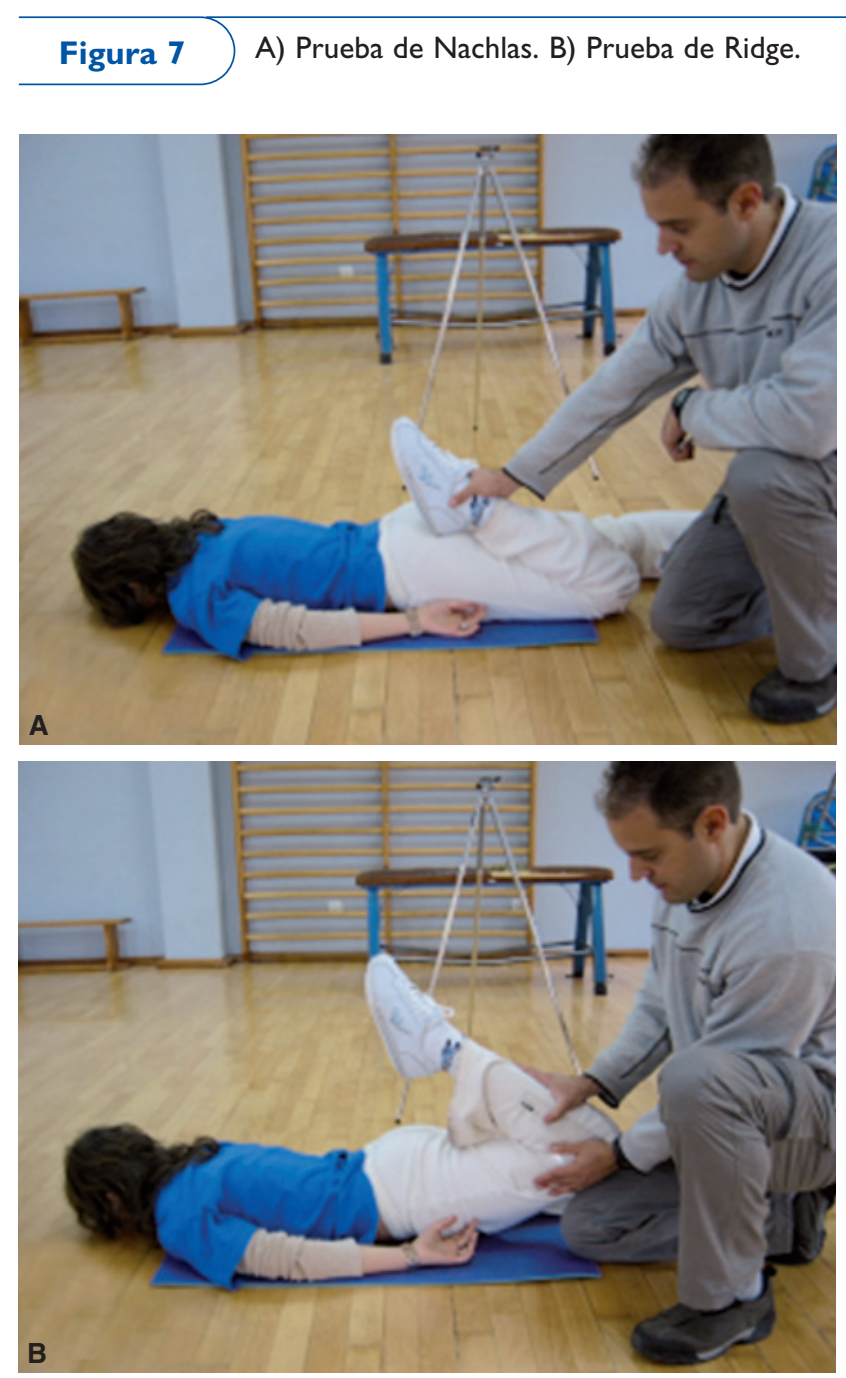

130 y $140^{\circ}$ (fig. 7 A). Si la prueba es positiva, se utiliza la prueba que describe Park Ridge (fig. 7B), donde con una extensión previa de la cadera, un ángulo de $120^{\circ}$ se considera normal ${ }^{12}$.

\section{Prueba del pectoral $(\mathrm{PP})^{9}$}

- En bipedestación, el sujeto, de frente a la pared, eleva el brazo del mismo lado que el pectoral medido de forma que todo él quede paralelo al suelo y apoyado a la pared; entonces trata de llevar el hombro del lado contrario lo más atrás posible rotando el tronco, sin separar el brazo de la pared. Se mide el ángulo que forma el brazo con la espalda, tomando como origen el acromion, siendo uno de los lados del ángulo el brazo, y el otro, la línea que describen las tuberosidades acromiales del hombro derecho e izquierdo No superar los $90^{\circ}$ implica una deficiente flexibilidad de los grupos musculares implicados (fig. 8). 


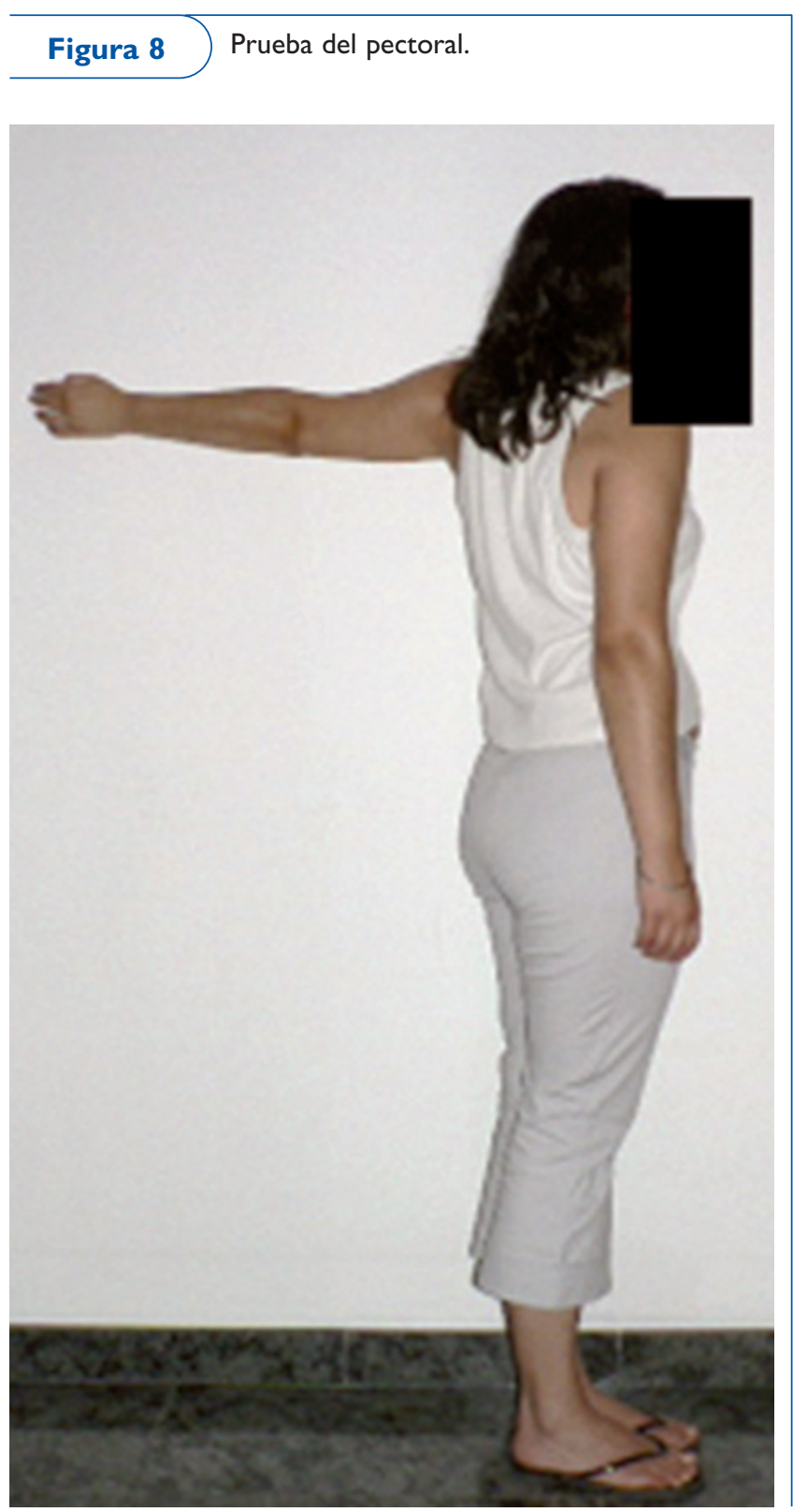

\section{Resultados}

Destaca el alto porcentaje de acortamientos y de flexibilidad reducida en los sujetos estudiados. Los resultados son peores a medida que el sujeto es mayor en edad. Los picos máximos de acortamientos se logran a diferentes edades, y en la mayoría de ellos se alcanzan en la última franja de edad, entre los 15 y los 17 años. Los datos en relación a los picos máximos en las diferentes pruebas son los siguientes (tabla 1).

- En la PK, o prueba de Kendall, se aprecian los mayores índices de acortamientos a los 16 y 17 años en los varones y a los 15 años en las mujeres, alcanzándose porcentajes del 93,8$91,7 \%$ (lado derecho e izquierdo respectivamente) en varones y del $76,5 \%$ en mujeres.

- En el DP se obtienen los porcentajes más elevados a los 7 años, con el $82,4 \%$ para el lado derecho y el $64,7 \%$ para el lado izquierdo en los varones, y en las mujeres a los 11 años en el lado derecho y a los 8 años en el izquierdo (65-50\%).

- En la prueba de FCRE, la edad en la que aparecen mayores porcentajes de acortamientos en los varones es a los 14 años (55$50 \%$ ) y en las mujeres a los 12 y 16 años en el lado derecho (15 y $14,3 \%$ ) y a los 8 y 15 años en el lado izquierdo (12,5 y 11,8\%).

- En la prueba PTh es a partir de los 8 años cuando se incrementan los porcentajes de acortamientos. En los varones el valor máximo se alcanza a los 17 años en el lado derecho y a los 15 años en el izquierdo (58,3-57,1\%). En las mujeres, a los 10 años el 50\% de los sujetos tienen acortamientos en el lado derecho y a los 16 años el 42,9\% en el lado izquierdo. En el apartado de la PTh que mide posibles acortamientos en el recto anterior, los porcentajes mayoritarios en los varones aparecen a los 16 años en el lado derecho y a los 17 años en el izquierdo (31,3-58,3\%). En las mujeres, a los 17 años en el lado derecho y a los 10 años en el izquierdo (23,1-22,2\%).

- En los RCE, los acortamientos se acentúan a los 15-16 y 17 años (42,9-50-50\%) en los varones en el lado derecho y a los 15 años en el lado izquierdo (50\%). En las mujeres, a la edad de 13 años $(28,6-32,1 \%)$ se alcanzan mayores porcentajes de acortamientos.

- En los RCI, en los varones a los 15 y 17 años en el lado derecho y a los 16 años en el izquierdo (64,3-75-56,3\%). En las mujeres, los porcentajes más elevados se alcanzan a los $13 \mathrm{y}$ 17 años en el lado derecho $(35,7$ y $38,5 \%)$ y a los 17 años en el izquierdo (46,2\%).

- En la prueba de AC, en los varones estos porcentajes se alcanzan a los 15 años en el lado derecho (50\%) y a los 16 años en el izquierdo (75\%). En las mujeres, en el lado derecho es a los 11 años (50\%), y en el lado izquierdo a los 15 años (64,17\%).

- En la prueba de EFP, en los varones en el lado derecho a los 9 años se alcanzan porcentajes del 39,1\% y en el lado izquierdo, a los 10 años se alcanzan los mayores porcentajes de acortamientos, con el 53,8\%. En los sujetos mujeres, en el lado derecho a los 7 años se alcanza el 38,5\% y en el lado izquierdo a los 8 y a los 12 años se alcanza el $50 \%$.

- En las diferentes pruebas realizadas en el cuádriceps, los resultados tanto en la $\mathrm{PN}$ como en la PR son muy similares entre el lado derecho y el izquierdo. Las mayores variaciones se producen entre sexos. En los sujetos varones, en la primera parte de la prueba (intentar contactar el talón con el glúteo) los 
TRABAJOS ORIGINALES

Tabla I Picos máximos de acortamientos por edad y sexo

\begin{tabular}{|c|c|c|c|c|}
\hline \multirow[t]{2}{*}{ Edad (años) } & \multicolumn{2}{|c|}{ Varones } & \multicolumn{2}{|c|}{ Mujeres } \\
\hline & Prueba & Lado y porcentaje & Prueba & Lado y porcentaje \\
\hline \multirow[t]{2}{*}{7} & DP & D $82,4 \%$ & EFP & D $38,5 \%$ \\
\hline & & $164,7 \%$ & & \\
\hline \multirow[t]{2}{*}{8} & & & DP & $150 \%$ \\
\hline & & & EFP & $150 \%$ \\
\hline 9 & EFP & D $39,1 \%$ & FCRE & $111,8 \%$ \\
\hline \multirow[t]{2}{*}{10} & EFP & $153,8 \%$ & PTh & D $50 \%$ \\
\hline & & & Recto ant. (PTh) & I $22,2 \%$ \\
\hline \multirow[t]{3}{*}{11} & & & DP & D $65 \%$ \\
\hline & & & $A C$ & D $50 \%$ \\
\hline & & & $\mathrm{PN}$ & D $60 \%$ \\
\hline \multirow[t]{2}{*}{12} & & & FCRE & D I5\% \\
\hline & & & EFP & $150 \%$ \\
\hline \multirow[t]{4}{*}{13} & & & EFP & D $28,6 \%$ \\
\hline & & & $\mathrm{RCE}$ & $132,1 \%$ \\
\hline & & & $\mathrm{RCl}$ & D $35,7 \%$ \\
\hline & & & PN & $160 \%$ \\
\hline \multirow[t]{4}{*}{14} & FCRE & D $55 \%$ & PP & $150 \%$ \\
\hline & & $150 \%$ & & \\
\hline & PP & D $90 \%$ & & \\
\hline & & $180 \%$ & & \\
\hline \multirow[t]{4}{*}{15} & PTh & I $57,1 \%$ & PK & $76,50 \%$ \\
\hline & $\mathrm{RCE}$ & $150 \%$ & FCRE & $112,5 \%$ \\
\hline & $A C$ & D $50 \%$ & $A C$ & $164,7 \%$ \\
\hline & & & PP & D $58,8 \%$ \\
\hline \multirow[t]{5}{*}{16} & PK & $93,80 \%$ & FCRE & D $14,3 \%$ \\
\hline & $\mathrm{RCE}$ & D $50 \%$ & & \\
\hline & $\mathrm{RCl}$ & I $56,3 \%$ & & \\
\hline & Recto ant. (PTh) & D $31,3 \%$ & PTh & | $42,9 \%$ \\
\hline & $A C$ & $175 \%$ & & \\
\hline \multirow[t]{5}{*}{17} & PK & $91,70 \%$ & Recto ant. (PTh) & D $23,1 \%$ \\
\hline & PTh & D $58,3 \%$ & $\mathrm{RCl}$ & D $38,5 \%$ \\
\hline & $\mathrm{RCl}$ & D $75 \%$ & $\mathrm{RCl}$ & | $46,2 \%$ \\
\hline & $\mathrm{PN}$ & $83,30 \%$ & & \\
\hline & Recto ant. (PTh) & I $58,3 \%$ & & \\
\hline
\end{tabular}

AC: aductores de cadera; D: lado derecho; DP: diagonal posterior; EFP: elongación de los flexores plantares; FCRE: flexión de cadera con rodilla en extensión; I: lado izquierdo; PK: prueba de Kendall; PN: prueba de Nachlas; PP: prueba del pectoral; PTh: prueba de Thomas; RCE: rotadores de cadera externos; RCl: rotadores de cadera internos. 


\section{TRABAJOS ORIGINALES}

mayores porcentajes de acortamiento se alcanzan a los 17 años $(83,3-83,3 \%)$. En la mujer se alcanza en el lado derecho a los 11 años y en el lado izquierdo a los 13 años (60-64,3\%).

- En la PP, los mayores porcentajes de acortamiento se alcanzan en los varones a los 14 años $(90-80 \%)$ y en las mujeres a los 15 años en el lado derecho y a los 14 años en el lado izquierdo (58,8-50\%).

Las medias obtenidas en las diferentes pruebas realizadas confirman que los resultados son mejores en las mujeres (tabla 2).

Tabla 2 Medias obtenidas en las diferentes pruebas realizadas. Valores expresados en grados

\begin{tabular}{|c|c|c|c|c|c|c|c|c|c|}
\hline & & $\mathbf{N}$ & Media & Desviación típica & & & $\mathbf{N}$ & Media & Desviación típica \\
\hline \multirow[t]{3}{*}{ PKD } & Varón & 213 & 175,60 & 6,60 & ACD & VARÓN & 213 & 43,61 & 8,52 \\
\hline & Mujer & 207 & 175,73 & 5,99 & & Mujer & 207 & 45,29 & 10,62 \\
\hline & Total & 420 & 175,66 & 6,30 & & Total & 420 & 44,44 & 9,64 \\
\hline \multirow[t]{3}{*}{ PKI } & Varón & 213 & 175,76 & 6,28 & $\mathrm{ACl}$ & Varón & 213 & 40,77 & 9,11 \\
\hline & Mujer & 207 & 176,13 & 5,59 & & Mujer & 207 & 42,63 & 10,94 \\
\hline & Total & 420 & 175,94 & 5,95 & & Total & 420 & 41,69 & 10,08 \\
\hline \multirow[t]{3}{*}{ FCRED } & Varón & 213 & 83,09 & 10,57 & EFPD & Varón & 213 & 12,52 & 6,89 \\
\hline & Mujer & 207 & 92,57 & 12,32 & & Mujer & 207 & 11,86 & 7,23 \\
\hline & Total & 420 & 87,76 & 12,40 & & Total & 420 & 12,20 & 7,06 \\
\hline \multirow[t]{3}{*}{ FCREI } & Varón & 213 & 79,00 & 11,16 & EFPI & Varón & 213 & 12,35 & 7,17 \\
\hline & Mujer & 207 & 87,89 & 13,03 & & Mujer & 207 & 11,25 & 7,56 \\
\hline & Total & 420 & 83,38 & 12,90 & & Total & 420 & ||$, 8 \mid$ & 7,37 \\
\hline \multirow[t]{3}{*}{ PThD } & Varón & 213 & 107,40 & 12,44 & PND & Varón & 120 & 130,83 & 7,53 \\
\hline & Mujer & 207 & 107,46 & 12,13 & & Mujer & 81 & 132,58 & 8,33 \\
\hline & Total & 420 & 107,43 & 12,27 & & Total & 201 & $13 \mid, 53$ & 7,89 \\
\hline \multirow[t]{3}{*}{ PThl } & Varón & 213 & II 2,84 & 13,09 & PNI & Varón & 123 & 130,06 & 8,01 \\
\hline & Mujer & 207 & II 2,78 & 11,03 & & Mujer & 83 & $|3|, 3 \mid$ & 9,21 \\
\hline & Total & 420 & || $2,8 \mid$ & 12,10 & & Total & 206 & 130,56 & 8,52 \\
\hline \multirow[t]{3}{*}{ RCED } & Varón & 213 & 40,68 & 7,31 & PRD & Varón & 93 & 142,22 & 9,35 \\
\hline & Mujer & 207 & 44,22 & 7,55 & & Mujer & 127 & 143,57 & 8,76 \\
\hline & Total & 420 & 42,42 & 7,63 & & Total & 220 & 143,00 & 9,02 \\
\hline \multirow[t]{3}{*}{ RCEI } & Varón & 213 & 40,70 & 7,70 & PRI & Varón & 91 & 142,38 & 9,15 \\
\hline & Mujer & 207 & 45,14 & 8,23 & & Mujer & 125 & 143,03 & 8,67 \\
\hline & Total & 420 & 42,89 & 8,26 & & Total & 216 & 142,76 & 8,86 \\
\hline \multirow[t]{3}{*}{ RCID } & Varón & 213 & 36,98 & 6,86 & PPD & Varón & 213 & 91,69 & 14,55 \\
\hline & Mujer & 207 & 40,32 & 6,70 & & Mujer & 207 & 95,37 & 12,82 \\
\hline & Total & 420 & 38,63 & 6,98 & & Total & 420 & 93,50 & 13,83 \\
\hline \multirow[t]{3}{*}{ RCII } & Varón & 213 & 37,22 & 6,35 & PPI & Varón & 213 & 88,62 & 14,06 \\
\hline & Mujer & 207 & 38,97 & 5,92 & & Mujer & 207 & 92,18 & 12,82 \\
\hline & Total & 420 & 38,08 & 6,20 & & Total & 420 & 90,38 & 13,56 \\
\hline
\end{tabular}

AC: aductores de cadera; D final: lado dececho; EFP: elongación de los flexores plantes; FCRE: flexión de cadera con rodilla en extensión; I final: lado izquierdo; PK: prueba de Kendall; PN: prueba de Nachlas; PP: prueba del pectoral; PR: prueba de Ridge; PTh: prueba de Thomas; RCE: rotadores de cadera externos; RCl: rotadores de cadera inernos. 
TRABAJOS ORIGINALES

Figura 9

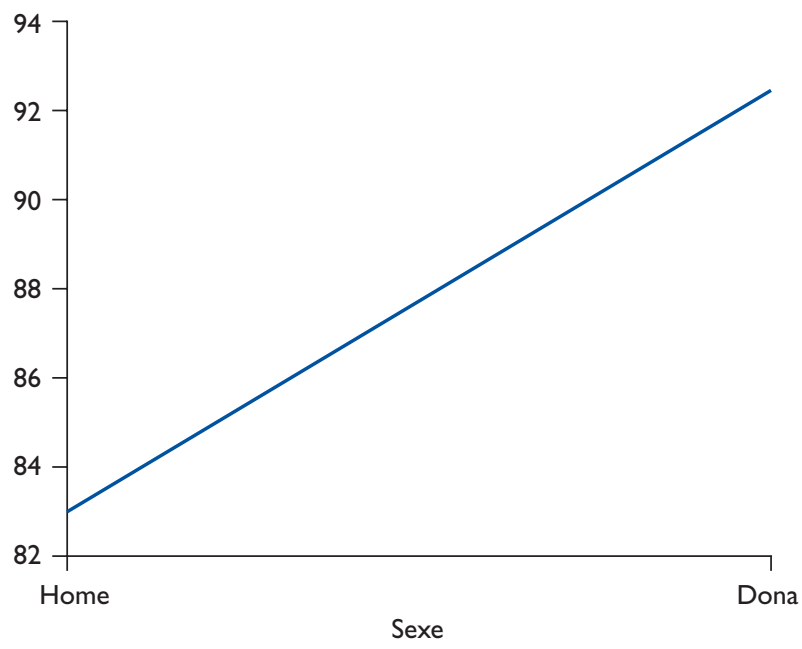

Existen diferencias significativas en las siguientes pruebas:

- FCRE derecha $(0,000)$ e izquierda $(0,000)$.

- RCE del lado derecho $(0,000)$ e izquierdo $(0,000)$.

- RCI del lado derecho $(0,000)$ e izquierdo $(0,000)$.

- Pectoral derecho $(0,007)$ e izquierdo $(0,006)$.

Las medias obtenidas en estas pruebas, dependiendo de si el sujeto es varón o mujer, fueron las siguientes:

- En la FCRE existen diferencias de casi $10^{\circ}$ en el lado derecho y cerca de los $9^{\circ}$ en el lado izquierdo. Los resultados son de 83,09 y $92,57^{\circ}$ en el lado derecho para varones y mujeres, respectivamente. En el lado izquierdo, los resultados obtenidos son de 79 y $87,89^{\circ}$ (fig. 9).

- Rotadores de cadera. En los RCE, las medias obtenidas en los varones fueron de 40,68 y 40,70 (lado derecho e izquierdo). En las mujeres, en el lado derecho obtienen una media de $44,22^{\circ}$, y en el lado izquierdo, de $45,14^{\circ}$. En los RCI, los varones alcanzan una media para el lado derecho e izquierdo, respectivamente, de 36,98 y $37,22^{\circ}$. En las mujeres, estas medias suben hasta $40,32^{\circ}$ para el lado derecho y $38,97^{\circ}$ para el lado izquierdo (fig. 10).

- En la PP, las medias obtenidas para el lado derecho en varones y mujeres, respectivamente, fueron de 91,69 y 95,37\%. En el lado izquierdo los resultados fueron de 88,62 y $92,18^{\circ}$ (fig. 11).

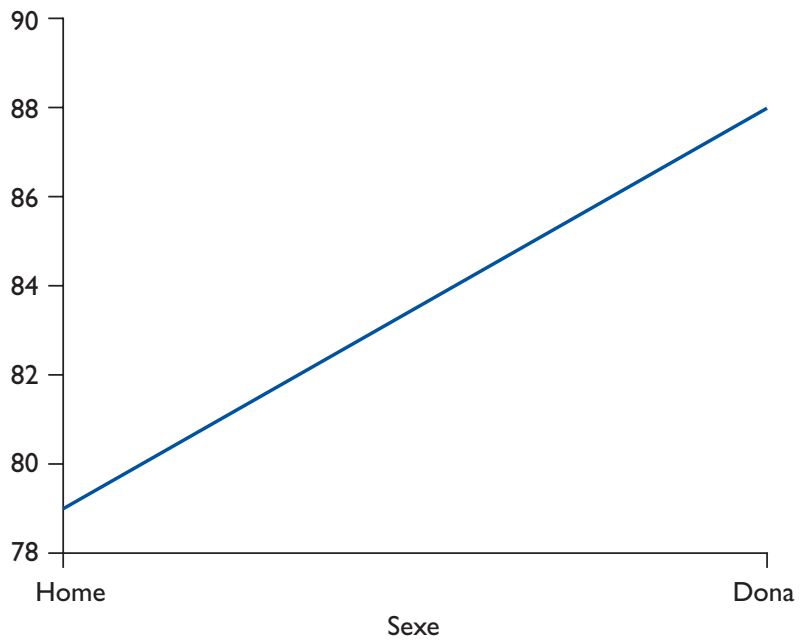

\section{Discusión}

A lo largo de la vida escolar, desde que ingresan en un centro educativo de educación primaria y hasta que abandonan sus estudios, ya sean obligatorios o se extiendan hasta el campo universitario, se producen adaptaciones musculares determinadas por el estilo de vida del escolar en lo que se refiere a su acondicionamiento físico. Se somete a los alumnos a largas estancias en posición sedente o de inactividad y a cargar pesadas mochilas en sus desplazamientos. Esta situación provoca la adaptación de su organismo a los requerimientos a los que se somete. La musculatura más implicada se desarrolla en mayor medida que la que no es solicitada para las distintas funciones cotidianas del escolar.

Así por ejemplo, permanecer sentado durante largas horas en el centro escolar en rígidas sillas construidas para provocar un estado de atención supondrá el acortamiento de determinados grupos musculares y el agotamiento y finalmente la distensión de otros. Este acortamiento va a ser provocado en la musculatura flexora de determinadas articulaciones implicadas, y la distensión es ocasionada en aquellos grupos musculares antagonistas a los primeros ${ }^{14,15}$.

Esta postura ha de ser mantenida durante mucho tiempo, llegándose hasta las $6 \mathrm{~h}$ diarias ${ }^{16,17}$, lo cual conlleva una serie de modificaciones, como ${ }^{15}$ :

- Acortamiento de la musculatura flexora del tobillo (tibial y peroneo anterior), más acusado con angulaciones de tobillo inferiores a $90^{\circ}$. 
TRABAJOS ORIGINALES

Figura 10 Medias obtenidas en varones y mujeres en las pruebas de RCE y RCl. RCE: rotadores de cadera externos; $\mathrm{RCl}$ : rotadores de cadera internos.
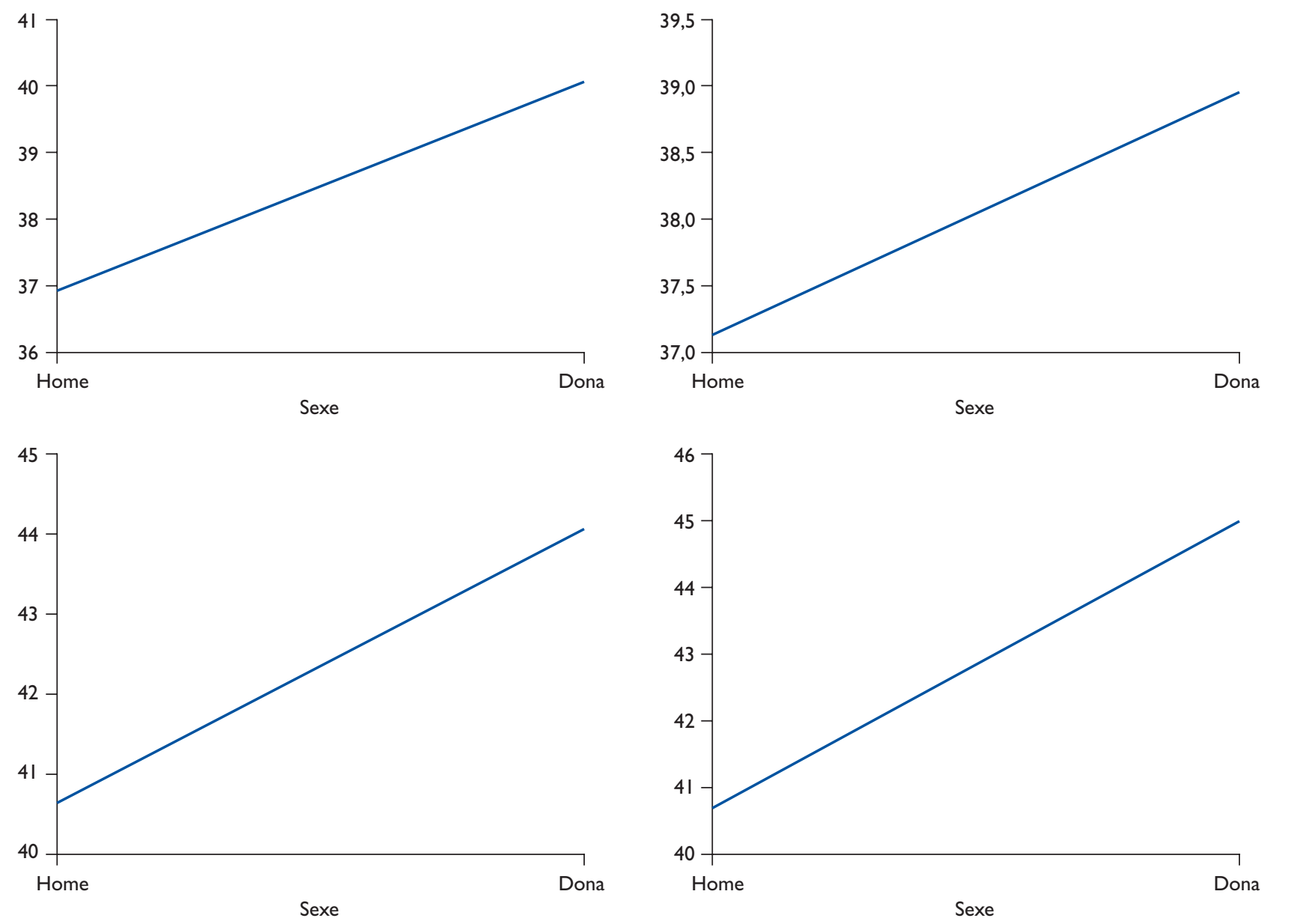

Figura II Medias obtenidas en la prueba PP, en varones y mujeres. PP: prueba de pectoral.
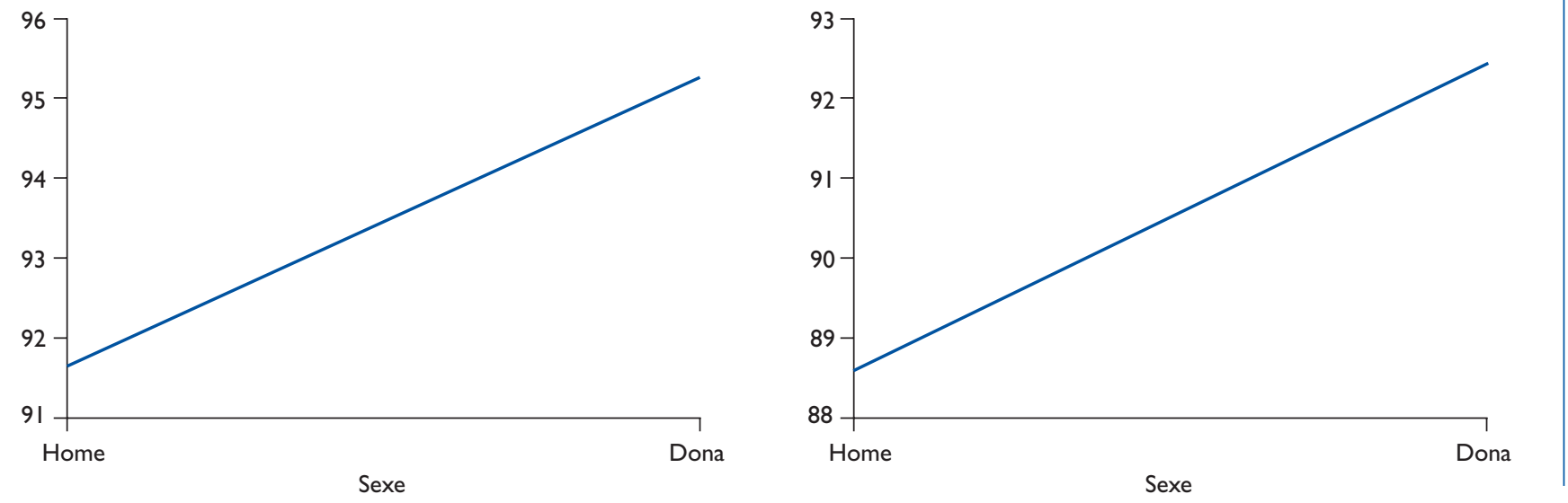
- Acortamiento de la musculatura flexora de la rodilla (isquiotibiales). Aunque se debe considerar que los isquiotibiales son, además de flexores de rodilla, extensores de cadera, por lo que el análisis biomecánico es más complejo. Podría pensarse que la elongación en la cadera compensa el acortamiento de la rodilla y equilibra su longitud; pero no es tal, ya que el alargamiento en la cadera es muy inferior en su brazo de palanca que el acortamiento producido en la rodilla. Los isquiotibiales tienen una amplitud comprendida entre los 130 y los $145^{\circ} \mathrm{de}$ arco en la rodilla y tan sólo de 15 a $30^{\circ}$ de extensión en la cadera. Estos dos factores hacen que la posición sentada sea favorecedora del acortamiento ${ }^{18}$.

- Acortamiento de la musculatura flexora de la cadera (músculo psoas ilíaco). El psoas ilíaco es flexor de cadera, por lo tanto está en postura acortada cada vez que estamos en flexión de cadera. Los escolares adoptan esta postura durante la mayor parte del horario, lo cual les provoca lenta y persistentemente un acortamiento del psoas ilíaco.

- Acortamiento y sobrecarga de la musculatura extensora del cuello (fibras superiores del trapecio) para el mantenimiento del peso de la cabeza.

- Acortamiento de la zona pectoral (pectoral mayor) y disimetría derecha/izquierda de la articulación glenohumeral. La posición de escritura provoca, en caso de no disponer de un mobiliario adecuado, un acortamiento de la musculatura, en el lado predominante, del pectoral y de los elevadores de la escápula.

- Agotamiento de la musculatura erectora de la columna vertebral y, por lo tanto, adopción de una postura cifótica en unas ocasiones, y de apoyo isquiosacro en otras.

Como consecuencia de lo expuesto anteriormente, los músculos pierden su capacidad de relajación y se agotan todavía más, originándose así un círculo vicioso difícil de anular.

El número de investigaciones sobre la flexibilidad muscular de los niños en escuelas de educación primaria es escaso (Fernández, citado por Santonja ${ }^{5}$ ). En educación secundaria ocurre lo mismo. Por ello se hace necesaria la realización de un mayor número de investigaciones que incidan en el tratamiento de este contenido de forma más profunda en las programaciones didácticas llevadas a cabo en la asignatura de educación física.

Los resultados obtenidos en el estudio permiten afirmar que ya en edades tempranas (7 años) aparecen acortamientos en las diversas pruebas planteadas, y la prueba de la diagonal posterior muestra un porcentaje mayoritario. Este tipo de resultado es cuanto menos preocupante.

Una de las posibles soluciones sería la disminución de algunos ejercicios en las clases de educación física, en favor de otros menos tratados tradicionalmente, que permitan a los alumnos compensar su musculatura y desarrollarla equilibradamente. Debido al porcentaje de alumnos que no alcanzan los rangos considerados normales en las diversas pruebas propuestas, hay que plantearse qué tipo de ejercicios se incluirán en las programaciones didácticas de educación física. Estos contenidos erróneos, en forma de actividades físicas inadecuadas, pueden provocar malos hábitos en el alumno, desequilibrios musculares o incluso lesiones de la columna vertebral.

Otra solución sería aumentar el número de horas dedicadas a la educación física. Debido al escaso número de horas que la asignatura tiene en el currículum actual, es imposible realizar un trabajo compensatorio y de acondicionamiento físico adecuado.

La realización de estos test puede servir de herramienta válida para su utilización masiva por parte del profesorado que imparte la asignatura de educación física, tanto en educación primaria como en educación secundaria. Esto permitirá una valoración más amplia y global de la flexibilidad, la amplitud articular y la prevención de acortamientos y descompensaciones musculares.

Es conveniente realizar ejercicios de flexibilidad en las zonas tendentes al acortamiento. Estos ejercicios deben realizarse de forma masiva en edades tempranas, y sobre todo en las edades en las que aumenta el porcentaje de acortamientos.

\section{Bibliografía}

1. Zaragoza J, Serrano E, Generelo E. La medición de la condición física saludable: aplicación de la batería Eurofit para adultos [en línea]. 2004. Disponible en: http://www.efdeportes.com/ Revista Digital. Buenos Aires, Año 10, N. ${ }^{\circ}$ 68. [Consulta: 15/1/2006]

2. Mac Dougall JD, Wenger HA, Green HJ. Evaluación fisiológica del deportista. Badalona: Paidotribo; 1995.

3. Pila A. Preparación Física. Madrid: Pila Teleña; 1985.
4. Arregui JA, Martínez V. Estado actual de las investigaciones sobre la flexibilidad en la adolescencia. Revista Internacional de Medicina y Ciencias de la Actividad Física y Deporte. 2001;2.

5. Santonja F, Martínez-Moro I. Valoración medicodeportiva del escolar. Murcia: Universidad de Murcia; 1992.

6. Souchard PE. Stretching global activo (II). Badalona: Paidotribo; 1998. 
7. Liebenson C. Manual de rehabilitación de la columna vertebral. Badalona: Paidotribo; 1999.

8. González JL, Martínez J, Mora M, Salto G, Álvarez E. El dolor de espalda y los desequilibrios musculares. Int J Med Science Physic Activity Sport. 2004;13.

9. Daniels L, Worthingham C. Fisioterapia. Ejercicios correctivos de la alineación y función del cuerpo humano. Barcelona: Doyma; 1981.

10. Kendall HO, Kendall FP, Wadsworth GE. Músculos, pruebas y funciones. 2. ${ }^{\mathrm{a}}$ ed. Barcelona: JIMS; 1985.

11. Clarkson HM. Proceso evaluativo musculoesquelético: amplitud del movimiento articular y test manual de fuerza muscular. Badalona: Paidotribo; 2003.

12. Ridge IL. Manual of Orthopaedic Surgery. Chicago: American Orthopaedic Association; 1985.

13. Daza J. Test de movilidad articular y examen muscular de las extremidades. Buenos Aires: Ed. Médica Panamericana; 1996.
14. Morehouse LE, Miller AT. Fisiología del ejercicio. 9. ${ }^{\text {a }}$ ed. Buenos Aires: Editorial El Ateneo; 1986.

15. González Montesinos JL, et al. Trabajo de la flexibilidad en educación primaria y secundaria: prevención de descompensaciones musculares, Comunicación. II Congreso de Ciencias del Deporte. Madrid: INEF; 2002.

16. Vayer P, Duval A, Roncin C. Una ecología de la escuela. Barcelona: Paidós; 1993.

17. Ramos D, González JL, Mora J. Desarrollo y aplicación de un cuestionario sobre una población de ESO. Estudio de la posición sedente, transporte de mochila y posición acostado. Influencia sobre el dolor de espalda. Premio Real Academia de Medicina y Cirugía de Cádiz.; 2004.

18. Hidalgo E. Técnicas de stretching para la kinesiología. La educación física y las artes del movimiento. Santiago de Chile: Universidad de Chile; 1993. 